\title{
The radiation hardness of pulled silicon doped with germanium
}

\author{
A.P. Dolgolenko, G.P. Gaidar*, M.D. Varentsov, P.G. Litovchenko \\ Institute for Nuclear Research, NAS of Ukraine, \\ 47, prospect Nauky, 03680 Kyiv, Ukraine, fax: 380-44-5254463 \\ *Corresponding author: e-mail: gaidar@kinr.kiev.ua
}

\begin{abstract}
The radiation hardness of Czochralski grown $n$-type silicon samples doped with germanium $\left(N_{\mathrm{Ge}}=2 \times 10^{20} \mathrm{~cm}^{-3}\right)$ and without it was investigated after irradiation by fast neutrons. The dependence of the effective carrier concentration on fluence was described in the framework of Gossick's corrected model. It was found that doping the germanium impurity resulted in increase of $n$-Si radiation hardness. The isothermal annealing of $n$-Si $\langle\mathrm{Ge}\rangle$ after fluence $1.4 \times 10^{14} \mathrm{n}^{\mathrm{o}} \cdot \mathrm{cm}^{-2}$ was studied. It was shown that the annealing of defect clusters is caused by the annihilation of vacancy type defects in clusters with interstitial defects. For di-interstitial $\left(E_{1}=0.74 \mathrm{eV} ; v_{1}=3.5 \times 10^{6} \mathrm{~s}^{-1}\right)$, silicon interstitial atom $\left(E_{2}=0.91 \mathrm{eV} ; v_{2}=7 \times 10^{6} \mathrm{~s}^{-1}\right)$ and vacancy $\left(E_{V}=0.8 \mathrm{eV} ; \mathrm{v}=\right.$ $\left.1 \times 10^{7} \mathrm{~s}^{-1}\right)$ the migration energies and frequency factors were determined. During the storage at room temperature, the behaviour of defect levels $E_{c}-0.17 \mathrm{eV}$ and $E_{c}-0.078 \mathrm{eV}$ was studied in the samples of $\mathrm{Si}(\mathrm{DOFZ})$ and $\mathrm{Si}\langle\mathrm{Ge}\rangle$, correspondingly.
\end{abstract}

Keywords: silicon, neutron irradiation, radiation hardness, radiation defects, clusters.

Manuscript received 25.10.06; accepted for publication 26.03.07; published online 01.06.07.

\section{Introduction}

The enhancement of radiation hardness of semiconductor materials was and remains as an urgent problem in developments of devices that must properly work in the extremely intense radiation fields for a long term. One of the ways to solve this problem is introduction of effective recombination centres into semiconductor for primary radiation defects. In this aspect, doping the silicon crystals with isovalent impurities is of particular interest. Such impurity doping essentially reduces the generation rate of secondary radiation defects. The majority of models explaining the influence of isovalent impurities on processes of defect formation are based on the role of internal local lattice strains caused by a difference of covalent radii of both matrix and impurity atoms [1].

It was shown that after electron irradiation at low temperatures $(T \leq 90 \mathrm{~K})$, the presence of germanium dopants in silicon leads to the significant decrease of the formation efficiency of radiation defects [2]. Germanium atoms capture vacancies, and the germanium-vacancy centres $(\mathrm{Ge}-\mathrm{V})$ are formed. Such centres, according to [3], are thermally unstable and their annealing occurrs at about $240 \mathrm{~K}$. A new band in DLTS spectra after proton irradiation of $n$-Si $\langle\mathrm{Ge}\rangle$ samples at $30 \mathrm{~K}$ was attributed by authors [4] to the acceptor level of Ge-V $\left(E_{\mathrm{c}}-0.29 \mathrm{eV}\right)$.

The decrease of the formation efficiency of vacancy-related defects ( $\mathrm{VO}$ and $\mathrm{VO}_{2}$ ) in $\mathrm{Si}_{1-\mathrm{x}} \mathrm{Ge}_{\mathrm{x}}$ will take place even at $300 \mathrm{~K}$, when the $\mathrm{Ge}-\mathrm{V}$ centres are practically annealed in the course of irradiation by fast electrons with the energy $4 \mathrm{MeV}$ [5]. In [6], it was shown that the introduction rate for A-centres and divacancies in $\mathrm{Si}\langle\mathrm{Ge}\rangle$ samples was decreased by a factor of 2 to 3 in comparison with $\mathrm{Si}$ reference samples after electron irradiation at the room temperature. Authors explained this fact by the assumption that the germanium atoms are indirect recombination centres of primary radiation defects ( $\mathrm{V}$ and $\mathrm{I}$ ) in $\mathrm{Si}\langle\mathrm{Ge}\rangle$. Nevertheless, according to [6], the probability of vacancy capture by germanium atoms is approximately two orders less than that for oxygen.

In general, practically all the available literature concerns the influence of the electron irradiation on defect formation in $\mathrm{Si}\langle\mathrm{Ge}\rangle$. Therefore, main goals of the present work are: (i) to study the influence of neutron irradiation on the radiation hardness of silicon samples doped with germanium; (ii) to determine the nature of

\section{(C) 2007, V. Lashkaryov Institute of Semiconductor Physics, National Academy of Sciences of Ukraine}


some point defects; (iii) at various temperatures to investigate the isothermal annealing of irradiated $\mathrm{Si}\langle\mathrm{Ge}\rangle$ and to determine the main parameters of the annealing process resulting in defect clusters.

\section{Experiment}

Czochralski grown $(\mathrm{Cz})$ silicon samples $(n-\mathrm{Si}\langle\mathrm{Ge}\rangle)$ and standard $\mathrm{Cz}$-Si $n$-type ones without germanium dopant have been studied after irradiation by various fluences of fast-pile neutrons. The range of the initial concentrations of carriers was $(5.15 \ldots 5.5) \times 10^{14} \mathrm{~cm}^{-3}$ for both types of samples.

The single crystals of $n-\mathrm{Si}\langle\mathrm{Ge}\rangle$ (doped by phosphorus) were grown at the "Redmet-30" (TitaniumMagnesium Industrial Complex, Zaporozhye, Ukraine). The dislocation-free silicon was grown along a $\langle 100\rangle$ crystallographic direction with the uniform distribution of germanium. The concentrations of oxygen $\left(N_{\mathrm{O}_{i}} \approx\right.$ $\left.(5 \ldots 7) \times 10^{17} \mathrm{~cm}^{-3}\right)$ and carbon $\left(N_{\mathrm{C}} \approx 1 \times 10^{17} \mathrm{~cm}^{-3}\right)$ were determined by the infrared absorption bands $(9$ and $16 \mu \mathrm{m}$ ) using a Fourier-transform spectrometer at the room temperature. The concentration of carbon in $\mathrm{Si}\langle\mathrm{Ge}\rangle$ is two times higher in comparison to that in $\mathrm{Cz}-$ $\mathrm{Si}$. The additional enrichment with carbon was carried out to compensate the local lattice strains due to the presence of germanium atoms. The concentration of germanium $\left(N_{\mathrm{Ge}} \approx 2 \times 10^{20} \mathrm{~cm}^{-3}\right)$ was determined using the mass-spectrometer.

Also studied were the $n$-type Si (DOFZ) samples grown by the floating-zone technique (FZ) with subsequent diffusion oxygenation (DO) at $1200{ }^{\circ} \mathrm{C}$ during $48 \mathrm{~h}$. The carrier concentrations before irradiation were $7.46 \times 10^{12}$ and $1.3 \times 10^{12} \mathrm{~cm}^{-3}$.

The irradiation was performed in the horizontal channel of the water-water reactor (WWR-M) nearly to the room temperature $(287 \mathrm{~K})$. The $\mathrm{Si}\langle\mathrm{Ge}\rangle$ and $\mathrm{Cz}-\mathrm{Si}$ samples were irradiated by fluences from $7 \times 10^{12}$ up to $3 \times 10^{15} \mathrm{n}^{\circ} \cdot \mathrm{cm}^{-2}$. The Si (DOFZ) samples were irradiated by $4 \times 10^{12}$ and $5 \times 10^{12} \mathrm{n}^{\mathrm{o}} \cdot \mathrm{cm}^{-2}$ fluences. The flux of fast neutrons $\left(I_{f}\right)$ was determined using the ${ }^{32} \mathrm{~S}$ threshold detector (threshold energy is $E=0.95 \mathrm{MeV}$ ) with the accuracy of $10 \%$, and led to energy of neutrons, starting from $\sim 100 \mathrm{keV}$. The relation of the fluxes $I_{f}(0.1) / I_{f}(0.95)=0.986 / 0.690$ in the spectrum of fission neutrons was taken into account. The concentration of phosphorus that could be introduced into the sample due to the neutron transmutation at $10^{16} \mathrm{n}^{\mathrm{o}} \cdot \mathrm{cm}^{-2}$ fluence does not exceed $1 \%$ of the initial concentration of phosphorus dopant in the studied samples. After irradiation, the samples were stored at $293 \mathrm{~K}$ in a passive thermostat.

Measurements of the Hall coefficient and conductivity were carried out by using the Van der Pauw compensatory method on samples of $10 \times 10 \times 1 \mathrm{~mm}$ size with the accuracy of $3 \%$. Contacts were created with Al rubbed into the silicon-polished surface.

\section{Results}

In Fig. 1, the dependences of the effective carrier concentration $\left(n_{\text {eff }}\right)$ on the fluence of fast-pile neutrons for Cz-Si $n$-type samples doped with germanium $\left(N_{\mathrm{Ge}}=\right.$ $\left.=2 \times 10^{20} \mathrm{~cm}^{-3}\right)$ and without it are shown. The measurements were carried out at $293 \mathrm{~K}$.

The $n_{\text {eff }}$ temperature dependences after irradiation of $n$-Si $\langle\mathrm{Ge}\rangle$ and $n$-Si (DOFZ) samples by various fluences of fast neutrons are shown in Figs 2 and 3. After irradiation, the $n$-Si (DOFZ) samples were stored at $293 \mathrm{~K}$ for various retention intervals.

In Fig. 4, the dependences of carrier concentrations removed by defect clusters from the conduction band $\left(n_{\mathrm{cl}}\right)$ on the annealing duration $(t)$ are shown. The consecutive isothermal annealing of $\mathrm{Si}\langle\mathrm{Ge}\rangle$ originally irradiated by the fluence $1.4 \times 10^{14} \mathrm{n}^{\mathrm{o}} \cdot \mathrm{cm}^{-2}$ was studied at three temperatures $(293,353$ and $379 \mathrm{~K})$. Measurements were carried out during storage at $293 \mathrm{~K}$ after 2, 26 and 55 days. At 353 and $379 \mathrm{~K}$ the time intervals of annealing were 20, 100 and $300 \mathrm{~min}$.

\section{Discussion}

It is generally accepted that the radiation hardness of silicon is determined by fluence, when the material retains its electro-physical properties under nuclear irradiation. The dose dependence of the effective concentration ( $\left.n_{\text {eff }}\right)$ and mobility of charge carriers, obtained from measurements of the Hall constant and conductivity at the room temperature, are usually used to define the radiation hardness of silicon. In our investigation, the criterion of radiation hardness can be calculated on the base of the determination of the carrier removal rate from the conduction band by point defects and clusters [7]

$v_{e}=v_{p}(1-f)-n_{0} \frac{f}{\Phi}$,

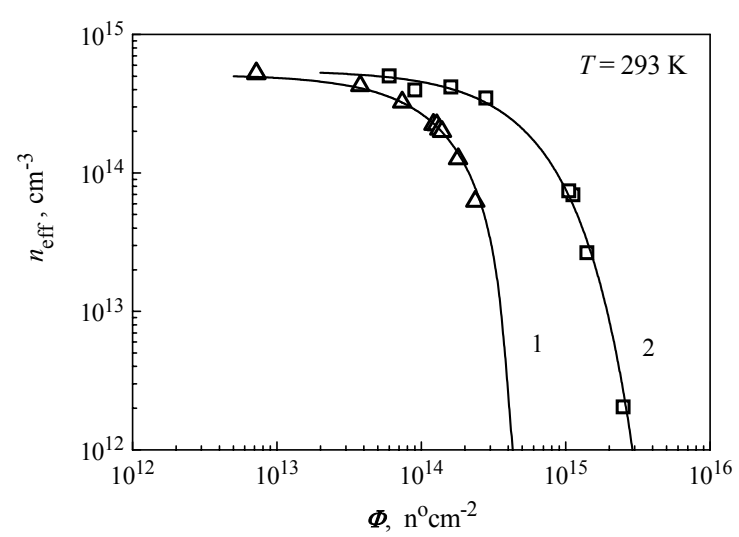

Fig. 1. Dependences of the effective carrier concentration $\left(n_{\text {eff }}\right)$ on the fluence of fast-pile neutrons $(\Phi)$ at $293 \mathrm{~K}$ in $\mathrm{Cz}-\mathrm{Si}$ samples: $1-n$-Si without $\mathrm{Ge}(\Delta) ; 2-n$-Si $\langle\mathrm{Ge}\rangle(\square)$. Triangles and squares denote the experimental data; solid lines - the theoretical calculations. 
where $v_{p}$ is the carrier removal rate by point defects; $v_{\mathrm{cl}}=-n_{0} f / \Phi$ is the carrier removal rate by defect clusters; $n_{0}$ is the carrier concentration before irradiation; $f(T, \Phi)=1-\exp (-\Sigma V(T) \Phi)$ is the volume fraction occupied by defect clusters at temperature $T$ after fluence of fast neutrons $\Phi[8] ; V(T)$ is the average volume of cluster [9]; $\Sigma=0.15 \mathrm{~cm}^{-1}$ is the macroscopic cross-section of the defect cluster creation [7].

In fact, from Eq. (1) the radiation hardness can be evaluated to a first approximation as the fluence $\left(\Phi_{\mathrm{H}}\right)$, at which the sample volume is totally occupied by clusters $(f=1)$. Then, from Eq. (1) one can obtain $\Phi_{\mathrm{H}}=n_{0} / v_{e}$.

Then the concentration of carriers $\left(n_{\mathrm{cl}}\right)$ removed by defect clusters from the conduction band is proportional to the volume fraction occupied by clusters $(f(T, \Phi))$, namely, $n_{\mathrm{cl}}(T, \Phi)=\mathrm{v}_{\mathrm{cl}} \Phi=n_{0} f(T, \Phi) . n_{\mathrm{eff}}$ can be defined as $n_{\text {eff }}=n_{0}+v_{e} \Phi$. However, we cannot use directly this formula, since the carrier removal rate by defects depends on $\Phi$. The analysis of Eq. (1) shows that with an increase of fluence the carrier removal rate $\left(v_{e}\right)$ decreases when increasing the volume fraction occupied by defect clusters. At the same time, fast neutrons form deep levels of defects such as divacancies, trivacancies in the conducting matrix. Therefore, with an increase of $\Phi$ the Fermi level shifts to the midgap, and consequently the carrier removal rate by point defects in the conducting matrix is also decreasing.

The $n_{\text {eff }}$ dependence on the fluence $\Phi$ and temperature $T$ can be written as [10]

$n_{\text {eff }}(T, \Phi)=n(T, \Phi)(1-f(T, \Phi))$,

where $n(T, \Phi)$ is the carrier concentration in the conducting matrix of $n$-Si.

Therefore, being based on Gossick's corrected model [11] and taking into account the capture of carriers at the deep levels of defects in the conducting matrix we may calculate $n_{\text {eff }}$ by using the equation [12]

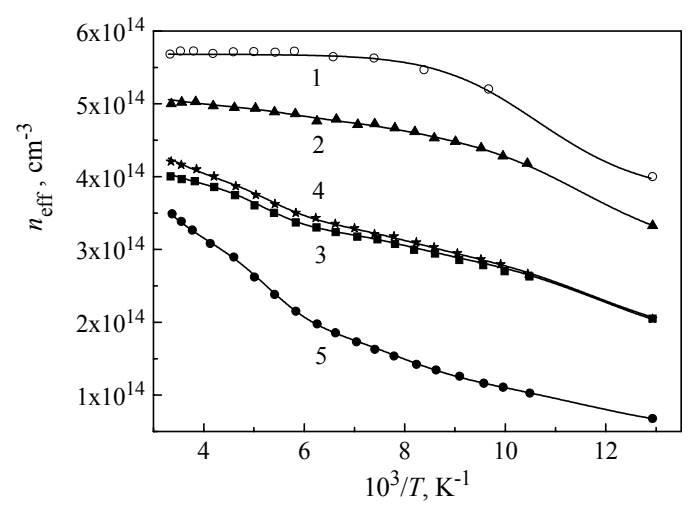

Fig. 2. Temperature dependences of the effective carrier concentration $\left(n_{\text {eff }}\right)$ for $n-\mathrm{Si}\langle\mathrm{Ge}\rangle$ before (1) and after irradiation by the following fluences of fast-pile neutrons: $2-3 \times 10^{13}$; $3-4.5 \times 10^{13} ; 4-8 \times 10^{13} ; 5-1.4 \times 10^{14} \mathrm{n}^{0} \cdot \mathrm{cm}^{-2}$. Geometrical figures denote the experimental data; solid lines - the theoretical calculations.

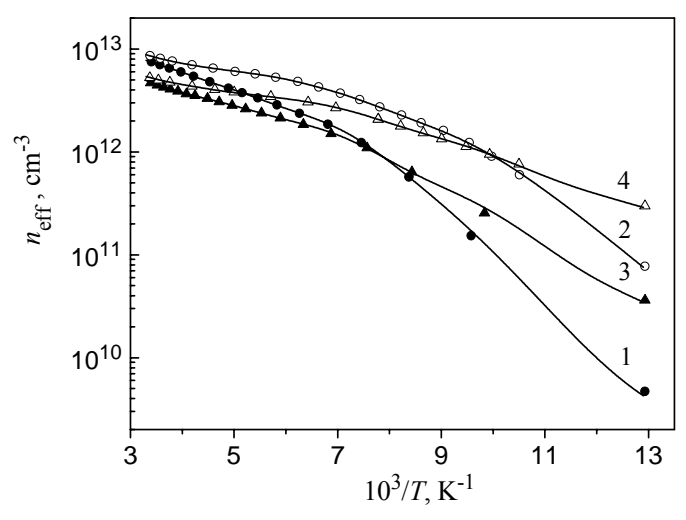

Fig. 3. Temperature dependences of the effective carrier concentration $\left(n_{\text {eff }}\right)$ for $n$-Si (DOFZ) after irradiation by two fluences of fast-pile neutrons: $5 \times 10^{12} \mathrm{n}^{0} \cdot \mathrm{cm}^{-2}(1,2-$ the storage time 2 and 90 days, respectively); $4 \times 10^{12} \mathrm{n}^{\mathrm{o}} \cdot \mathrm{cm}^{-2}$ (3,4 - the storage time 1 and 180 days, respectively). Geometrical figures denote the experimental data; solid lines the theoretical calculations.

$$
\begin{aligned}
& n_{\mathrm{eff}}(T, \Phi)=n(T, \Phi) \times \\
& \times \exp \left[-\frac{4 \pi \varepsilon \varepsilon_{0} \sum R_{1} \Phi}{N_{2}(T, \Phi) q^{2}}\left(\mu-k T \ln \frac{N_{\mathrm{c}}(T)}{N_{2}(T, \Phi)}\right)\right],
\end{aligned}
$$

where $N_{2}(T, \Phi)$ is the concentration of screening centres in space-charge regions (SCRs) of defect clusters; $\varepsilon, \varepsilon_{0}$ are the dielectric constants of material and vacuum, respectively; $\mu$ is the Fermi level position relative to the conduction band bottom in a cluster; $N_{\mathrm{c}}(T)$ is the effective state density in the conduction band. In more detail this technique is described in [13].

Let us consider phosphorus-doped $n$-Si with the carrier concentration $N_{d}\left(N_{d}<10^{15} \mathrm{~cm}^{-3}\right)$ and average degree of compensation by acceptors (boron) within the range from the room temperature down to liquid nitrogen one. Let neutrons create uniformly distributed point acceptor defects in $n$-Si volume (except for disordered regions) with the concentration $N_{a}<N_{d}$. Then, with increase of the sample temperature from $77 \mathrm{~K}$ the thermal excitation (ionization) of carriers from $E_{a}$ level gives some electron concentration in the conduction band both in the conducting matrix $n(T, \Phi)$ and in SCRs of defect clusters $N_{2}(T, \Phi)$

$$
\begin{aligned}
& n\left(T, \Phi, E_{a}\right)=\frac{1}{2}\left(N_{d}-\frac{N_{a}(\Phi)}{\lambda}-n_{11}\right) \times \\
& \times\left(\sqrt{1+\frac{4 N_{d} n_{11}}{\left(N_{d}-\frac{N_{a}(\Phi)}{\lambda}-n_{11}\right)^{2}}}+1\right), \\
& n_{11}=g N_{c}(T) \exp \left(-\frac{E_{a}}{\lambda k T}\right),
\end{aligned}
$$



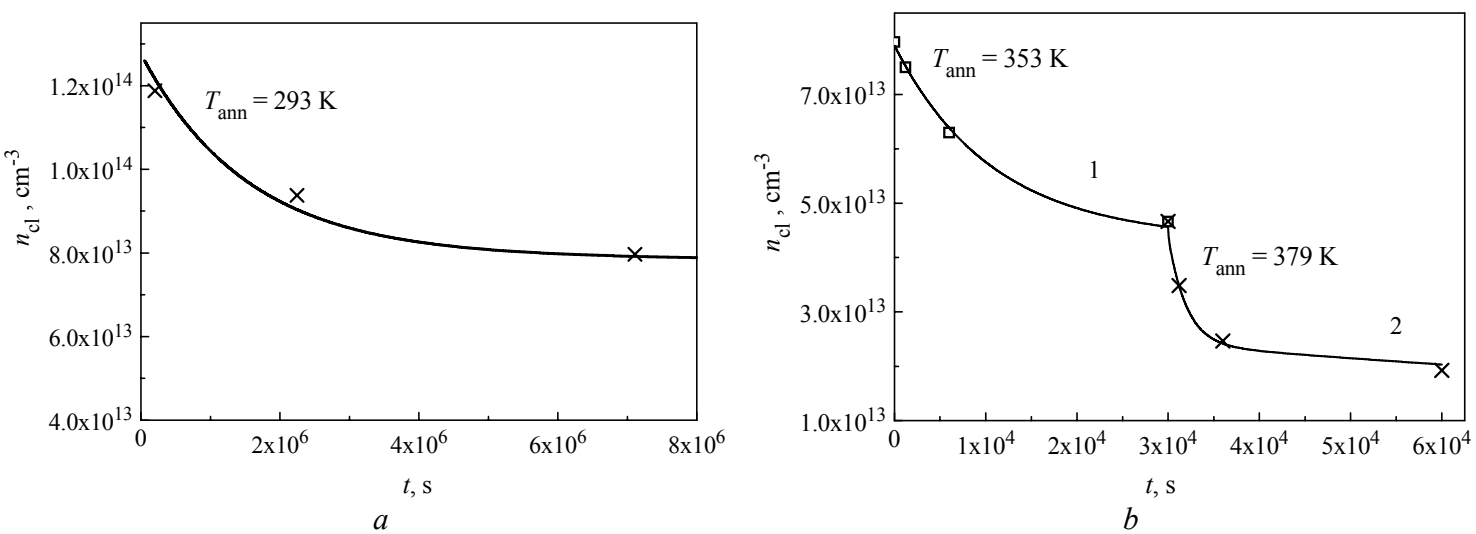

Fig. 4. Dependences of the carrier concentration removed by defect clusters $\left(n_{\mathrm{cl}}\right)$ on the annealing duration $(t)$ in $\mathrm{Si}\langle\mathrm{Ge}\rangle: a-$ at $293 \mathrm{~K} ; b-$ at $353 \mathrm{~K}(1)$ and $379 \mathrm{~K}(2)$. The initial fluence of fast-pile neutrons $(\Phi)$ was $1.4 \times 10^{14} \mathrm{n}^{\circ} \cdot \mathrm{cm}^{-2}$. Daggers and squares denote the experimental data; solid lines - the theoretical calculations.

where $g=2$ is the degeneration factor of the acceptor level; $N_{a}(\Phi)$ is the concentration of radiation-induced acceptor defects after fluence $\Phi ; n_{11}$ is the concentration of electrons in the $n$-Si conduction band, when the Fermi level coincides with the level $E_{a}$ and $E_{a} / \lambda$ one-level defect, which may be in the conducting matrix and in the SCR of defect cluster, respectively.

The bend of energy zones in the cluster at the Debye screening distance with a temperature decrease leads to the spatially non-uniform capture of the electron on acceptor defects according to (3), (4). If the acceptor defect is located in the conducting matrix, then $\lambda=1$, and in case of its being in the SCR of clusters $\lambda=1.5$.

In the absence of statistical interaction between levels of radiation defects the carrier concentration in the conducting matrix of $n$-Si can be determined, if the total carrier concentration $\sum_{1}^{4} n_{i}\left(T, \Phi, E_{i}\right)$ (when used the four-level system) is taken into account. Such carrier concentration $n_{\mathrm{i}}\left(T, \Phi, E_{i}\right)$ in the conduction band is produced by ionization of acceptor levels $E_{i}$, namely, Acentres as well as levels of divacancies, when the temperature $(T)$ or fluence $(\Phi)$ changes. Then in the conducting matrix of samples the concentration of carriers $n(T, \Phi)$ in the conduction band of $n$-Si is equal:

$n(T, \Phi)=\sum_{1}^{4} n_{i}\left(T, \Phi, E_{i}\right)-3 N_{d}+2 N_{a}(\Phi)+N_{a 1}$,

where $n_{i}\left(T, \Phi, E_{i}\right)$ is defined by Eq. (4) for $\lambda=1(i=1 \ldots 4)$.

The concentration of screening centres in SCRs of defect clusters is similarly given by

$$
\begin{aligned}
& N_{2}(T, \Phi)=\sum_{1}^{4} N_{2 i}\left(T, \Phi, E_{i}\right)-3 N_{d}+ \\
& +\frac{2 N_{a}(\Phi)}{\lambda}+\frac{N_{a 1}(\Phi)}{\lambda},
\end{aligned}
$$

where $N_{2 i}\left(T, \Phi, E_{i}\right)$ is defined by Eq. (4) for $\lambda=1.5(i=1 \ldots 4)$. Here $N_{a}(\Phi), \quad N_{a 1}(\Phi)$ are the concentrations of the second and third acceptor levels, respectively.

In the case, when we used the three-level system, the calculations were carried out in accordance with Eqs (9) and (10) in [12].

\subsection{Dose dependences of the effective concentration of carriers}

The calculation of $n_{\text {eff }}$ was carried out in the framework of Gossick's model [9] with account for the recharge of defects in the SCR of defect clusters. In the improved model of defect clusters, it was supposed that in intrinsic silicon the Fermi level is situated at the neutral level of the divacancy $\left(E_{v}+0.52 \mathrm{eV}\right.$, in our estimations). When a free electron is captured, the energy of $\mathrm{V}_{2}$ centre will increase by $0.165 \mathrm{eV}$ [13]. Therefore, with increasing the dopant concentration the Fermi level in cluster will be shifted to the conduction band. Thus, in our samples, as it was calculated in [7], the Fermi level position in clusters is $\mu=E_{c}-0.465 \mathrm{eV}$.

Based on techniques described in $[7,11]$, the calculation of the average radius of defect clusters $\left(R_{1}\right)$ was performed. It was obtained that $R_{1}$ decreases from $70 \AA$ in $\mathrm{Cz}-\mathrm{Si}$ down to $40 \AA$ in $\mathrm{Si}\langle\mathrm{Ge}\rangle$. Such decrease can be explained in the following way. It is known that under the irradiation by fast neutrons, the strong local heating a sample in sites of the cascade formation of displaced atoms occurs and leads to the annealing of radiation defects. The discrepancy of covalent radii of $\mathrm{Si}$ and Ge leads to the anharmonicity of lattice atom oscillations in $\mathrm{Si}\langle\mathrm{Ge}\rangle$ samples. As a result, the thermal conductivity is decreased and the conservation time of the local temperature is increased. In its turn, this can lead to more effective annealing of defects in clusters and to decreasing $R_{1}$. But other mechanism of the influence (for example, the deformation mechanism) of 
germanium atoms on parameters of defect clusters can be also possible.

At the room temperature the deep acceptor levels of radiation defects are usually responsible for the removal of electrons from the conduction band of $n$-Si. Divacancies $\left(\mathrm{V}_{2}\right)$ and trivacancies $\left(\mathrm{V}_{3}\right)$, which were introduced into $n$-Si with a high rate [14], are characterized by deep levels $\mathrm{V}_{2}^{-}\left(E_{c}-0.42 \mathrm{eV}\right)$ and $\mathrm{V}_{3}^{-}\left(E_{c}-0.49 \mathrm{eV}\right)$ in the forbidden band. According to [15], in $\mathrm{Cz}-\mathrm{Si}$ the $\left(E_{c}-0.45 \mathrm{eV}\right)$ slope describes the combined effect of acceptor levels of divacancy and trivacancy in the conducting matrix. To theoretically describe the experimental data obtained (Fig. 1), it is necessary to use the various introduction rates $(\vartheta)$ of the $\left(E_{\mathrm{c}}-0.45 \mathrm{eV}\right)$ averaged level, namely, $\vartheta=1.3 \mathrm{~cm}^{-1}$ in $\mathrm{Cz}-\mathrm{Si}$ and $\vartheta=0.1 \mathrm{~cm}^{-1}$ in $\mathrm{Si}\langle\mathrm{Ge}\rangle$. Thus, the presence of germanium atoms in $\mathrm{Cz}-\mathrm{Si}$ reduces the introduction rate of deep levels of vacancy type defects in the conducting matrix of the sample approximately by the factor of 13. We suppose that Ge atoms are the centres of the Frenkel pairs annihilation. As known [16], at the room temperature the Frenkel pairs can exist for several hours, while Ge-V is quickly annealed [6] and cannot be considered as the indirect recombination centre. Besides, the probability to capture a vacancy by germanium is very small and does not explain the decrease of the introduction rate of vacancy defects. Thus, the ability of Ge atoms to serve as the recombination centres of the Frenkel pairs is explained by the decrease of the introduction rate not only of A centres and divacancies, but also of trivacancies in the conducting matrix of $\mathrm{Si}\langle\mathrm{Ge}\rangle$.

The acceptor type defects, created by irradiation both in the SCRs of clusters and the conducting matrix, will define the carrier removal rate in the expression $\Phi_{\mathrm{H}}=n_{0} / v_{e}$. Then, we obtained the values of fluences $\Phi_{\mathrm{H}}=5.15 \times 10^{14} / 1.3 \approx 4 \times 10^{14} \mathrm{n}^{\mathrm{o}} \cdot \mathrm{cm}^{-2}$ and $\Phi_{\mathrm{H}}=$ $5.5 \times 10^{14} / 0.1 \approx 5.5 \times 10^{15} \mathrm{n}^{\mathrm{o}} \cdot \mathrm{cm}^{-2}$ for $\mathrm{Cz}-\mathrm{Si}$ and $\mathrm{Si}\langle\mathrm{Ge}\rangle$ samples, correspondingly. Therefore, the ratio of fluences for $\mathrm{Si}\langle\mathrm{Ge}\rangle$ and $\mathrm{Cz}-\mathrm{Si}$ is close to 13.8. The estimation of $\Phi_{\mathrm{H}}$ from Fig. 1 will understate this ratio because the neutron fluence was too low to reach the $\mathrm{n} \rightarrow \mathrm{p}$ conversion, which is usually used to determine the radiation hardness. In our opinion, it is the introduction rate of point defects in the conducting matrix that will determine the $n \rightarrow p$ conversion. However, even for fluences used in our experiments, the experiment shows that the doping with germanium increases the radiation hardness of silicon.

\subsection{Temperature dependences of the effective concentration of carriers}

The theoretical calculations of the temperature dependences of $n_{\text {eff }}$ shown in Fig. 2 were carried out in the framework of Gossick's corrected model [11], according to Eqs (3)-(6), for $n$-Si $\langle\mathrm{Ge}\rangle$ samples with a
Table 1. The concentrations $\left(N_{a i}\right)$ and energies $\left(E_{a i}\right)$ of acceptor levels in $n$-Si $\langle\mathbf{G e}\rangle$ irradiated by the fluence $\Phi$ of fast neutrons.

\begin{tabular}{|c|l|l|l|l|}
\hline $\begin{array}{c}\Phi, \\
\mathrm{n}^{\mathrm{o}} \cdot \mathrm{cm}^{-2}\end{array}$ & $N_{0}, \mathrm{~cm}^{-3}$ & $N_{a i}, \mathrm{~cm}^{-3}$ & $\begin{array}{c}E_{\mathrm{c}}-E_{a i}, \\
\mathrm{eV}\end{array}$ & $R_{1}, \AA$ \\
\hline 0 & $5.68 \times 10^{14}$ & $1.9 \times 10^{14}$ & 0.078 & 0 \\
\hline $3 \times 10^{13}$ & $5.23 \times 10^{14}$ & $0.13 \times 10^{14}$ & 0.16 & 28 \\
& & $0.18 \times 10^{14}$ & 0.11 & \\
& & $1.70 \times 10^{14}$ & 0.074 & \\
\hline $4.5 \times 10^{13}$ & $4.80 \times 10^{14}$ & $0.54 \times 10^{14}$ & 0.183 & 40 \\
& & $0.3 \times 10^{14}$ & 0.112 & \\
& & $1.4 \times 10^{14}$ & 0.074 & \\
\hline $8 \times 10^{13}$ & $5.15 \times 10^{14}$ & $0.5 \times 10^{14}$ & 0.175 & 40 \\
& & $0.3 \times 10^{14}$ & 0.11 & \\
& & $1.5 \times 10^{14}$ & 0.074 & \\
\hline $1.4 \times 10^{14}$ & $5.50 \times 10^{14}$ & $1.4 \times 10^{14}$ & 0.18 & 40 \\
& & $0.7 \times 10^{14}$ & 0.115 & \\
& & $1.1 \times 10^{14}$ & 0.078 & \\
\hline
\end{tabular}

carrier concentration $\left(N_{0}\right)$ before irradiation. Parameters of calculations are listed in Table 1 with account for the Fermi level in clusters positioned at $\mu=$ $E_{c}-0.465 \mathrm{eV}$ [7].

The analysis has shown that Gossick's corrected model enables to determine the carrier concentration in $n$-Si conduction band after irradiation by fast-pile neutrons with the accuracy $10 \%$. This allowed estimate the accuracy in determination of the acceptor level position in the $n$-Si forbidden band (see Table 1) as close to $3 \%$.

Table 1 shows that the concentration of defects with the level position $E_{c}-0.076 \pm 0.002 \mathrm{eV}$ decreases with the increase of fluence, but for the defect with $E_{c}-0.112 \pm 0.002 \mathrm{eV}$ the inverse dependence takes place. Thus, their total concentration remains constant.

Carbon atoms are usually introduced additionally when doping the silicon with germanium to compensate the lattice deformation. The solubility limit of carbon in silicon is close to $10^{17} \mathrm{~cm}^{-3}$. The insoluble carbon atoms form complexes with oxygen such as $\mathrm{C}_{i} \mathrm{O}_{i}, \mathrm{C}_{2 i} \mathrm{O}_{2 i}$. It is known [17] that $\mathrm{C}_{i} \mathrm{O}_{i}$ complex is the electrically active donor with the level $E_{v}+0.36 \mathrm{eV}$. Concerning $\mathrm{C}_{2 i} \mathrm{O}_{2 i}$ we supposed that this complex is responsible for presence of the $E_{c}-0.078 \mathrm{eV}$ acceptor level in the $n-\mathrm{Si}\langle\mathrm{Ge}\rangle$ forbidden band before irradiation. After the storage of sample, irradiated by $1.4 \times 10^{14} \mathrm{n}^{\mathrm{o}} \cdot \mathrm{cm}^{-2}$ fluence, for three months at $293 \mathrm{~K}$ the acceptor levels of $E_{c}-0.133 \mathrm{eV}$ and $E_{c}-0.093 \mathrm{eV}$ are observed, but the concentration of the $E_{\mathrm{c}}-0.076 \mathrm{eV}$ level is decreased still by the factor of 2 .

In silicon single crystals, the fast neutrons create the defect clusters with a high local density of divacancies $\left(10^{20} \mathrm{~cm}^{-3}\right)$. In so doing the interstitial atoms come out on the sample surface or can be trapped by $\mathrm{C}_{i} \mathrm{C}_{s}, \mathrm{C}_{i} \mathrm{O}_{i}, \mathrm{C}_{2 i} \mathrm{O}_{2 i}, \mathrm{O}_{2 i}$ and $\mathrm{O}_{i}$ defects in the conducting matrix. When the annealing occurs, the interstitial and di-interstitial atoms of silicon annihilate (in the course of direct recombination) with vacancy defects of clusters, 
and single vacancies are released in the conduction matrix. In details and quantitatively, the annealing process will be considered further. These processes can be described by reactions

$$
\begin{array}{ll}
\mathrm{I}+\mathrm{C}_{2 i} \mathrm{O}_{2 i} \rightarrow \mathrm{IO}_{2 i} \mathrm{C}_{2 i} & \left(E_{c}-0.112 \mathrm{eV}\right) ; \\
\mathrm{V}+\mathrm{IO}_{2 i} \mathrm{C}_{2 i} \rightarrow \mathrm{C}_{2 i} \mathrm{O}_{2 i} & \left(E_{c}-0.076 \mathrm{eV}\right) ; \\
\mathrm{V}+\mathrm{C}_{2 i} \mathrm{O}_{2 i} \rightarrow \mathrm{C}_{i} \mathrm{C}_{s} \mathrm{O}_{2 i} & \left(E_{\mathrm{c}}-0.133 \mathrm{eV}, E_{c}-0.093 \mathrm{eV}\right) .
\end{array}
$$

Usually, the $E_{c}-0.11 \mathrm{eV}$ level is attributed to $\mathrm{IO}_{2 i}$ defect [18]. In [19], it was shown that in Czochralski grown silicon the metastable defect $\mathrm{C}_{i} \mathrm{C}_{s}$ is modified by oxygen. At that two acceptor levels, namely $E_{c}-0.13 \mathrm{eV}$ and $E_{c}-0.09 \mathrm{eV}$, with equal concentration are formed in the forbidden band. In $n$-Si $\langle\mathrm{Ge}\rangle$ the observed $E_{c}-$ $0.133 \mathrm{eV}$ and $E_{c}-0.093 \mathrm{eV}$ levels are also introduced in the equal concentrations. Thus, the oxygen dimers modify $\mathrm{C}_{i} \mathrm{C}_{s}$ defects.

The theoretical calculations of the temperature dependences of $n_{\text {eff }}$ for $n$-Si (DOFZ), presented in Fig. 3, were performed by the above technique. Parameters of calculations are listed in Table 2. The Fermi level in clusters can be calculated using the expression $\mu=E_{c}-$ $0.6+0.033 \log N_{0} / n_{\text {intr }}$, where $n_{\text {intr }}\left(10^{10} \mathrm{~cm}^{-3}\right)$ is the carrier concentration in the intrinsic silicon at $300 \mathrm{~K}$.

The Table 2 shows that the concentration of Acentres $\left(E_{c}-0.175 \mathrm{eV}\right)$ after irradiation was equal to the concentration of acceptor levels $E_{c}-0.17 \mathrm{eV}$ and $E_{c}-$ $0.204 \mathrm{eV}$ after the storage of the samples at the room temperature. Both $E_{c}-0.17 \mathrm{eV}$ [17] and $E_{c}-0.204 \mathrm{eV}$ [20] levels are attributed to A-centre. Furthermore, after keeping the samples at the room temperature the concentrations of $E_{c}-0.132 \mathrm{eV}$ and $E_{c}-0.09 \mathrm{eV}$ levels (equal) are increased. This growth is caused by the existence of $\mathrm{C}_{2 i} \mathrm{O}_{2 i}$ complexes in $n$ - $\mathrm{Si}$ (DOFZ) samples. The generation of vacancies in the conducting matrix of oxygenated $n$-Si occurs due to the annealing of small clusters. At the same time, the annealing of $n$-Si $\langle\mathrm{Ge}\rangle$ samples at $323 \mathrm{~K}$ does not lead to the appearance of the $E_{c}-0.204 \mathrm{eV}$ level.

Let's suppose that A-centres are formed not only via capturing the vacancies, but also the Frenkel pairs by oxygen. Then it is possible to assume that $E_{c}-0.17 \mathrm{eV}$ is the A-centre and $E_{c}-0.204 \mathrm{eV}$ is the A-centre modified by the interstitial silicon atom. A-centres created by the capture of the Frenkel pair immediately after irradiation have properties of the typical A-centre $\left(E_{\mathrm{c}}-0.17 \mathrm{eV}\right)$. The storage of the $\mathrm{n}$-Si (DOFZ) results in the modification of the A-centre by interstitial silicon atom. The model of this centre was considered in [21], where the presence of dopant atom increases the energy $\left(E_{c}-0.105 \mathrm{eV}\right)$ of A-centre. On the other hand, the addition of hydrogen atom decreases this energy $\left(E_{c}-\right.$ $0.325 \mathrm{eV}$ ) [22]. Since the germanium atom is a recombination centre for the Frenkel pairs, it becomes clear why after annealing the $E_{c}-0.204 \mathrm{eV}$ level is absent in $n$-Si $\langle\mathrm{Ge}\rangle$. In [23], the authors assumed that after modification of the A-centre by interstitial oxygen the electrically active defect is also formed in the $(0 /-)$ charged state.
Table 2. The concentrations $\left(N_{a i}\right)$ and energies $\left(E_{a i}\right)$ of defects in $n$-Si (DOFZ) after various fluences $(\Phi)$ of fast

\begin{tabular}{|c|c|c|c|c|c|}
\hline $\begin{array}{c}\Phi, \\
\mathrm{n}^{\mathrm{o}} \cdot \mathrm{cm}^{-2}\end{array}$ & $N_{0}, \mathrm{~cm}^{-3}$ & $\begin{array}{c}t, \\
\text { days }\end{array}$ & $N_{a i}, \mathrm{~cm}^{-3}$ & $\begin{array}{c}E_{c}-E_{a i}, \\
\mathrm{eV}\end{array}$ & $R_{1}, \AA$ \\
\hline \multirow[t]{2}{*}{$4 \times 10^{12}$} & $7.46 \times 10^{12}$ & 1 & $\begin{array}{l}1.3 \times 10^{12} \\
3.7 \times 10^{12} \\
1.5 \times 10^{12} \\
1.5 \times 10^{12}\end{array}$ & $\begin{array}{l}0.24 \\
0.175 \\
0.135 \\
0.09\end{array}$ & 50 \\
\hline & & 180 & $\begin{array}{l}0.4 \times 10^{12} \\
2.8 \times 10^{12} \\
2.0 \times 10^{12} \\
2.0 \times 10^{12}\end{array}$ & $\begin{array}{l}0.204 \\
0.17 \\
0.132 \\
0.09 \\
\end{array}$ & 30 \\
\hline \multirow[t]{2}{*}{$5 \times 10^{12}$} & $1.30 \times 10^{13}$ & 2 & $\begin{array}{l}1.16 \times 10^{12} \\
2.60 \times 10^{12} \\
5.04 \times 10^{12} \\
1.16 \times 10^{12}\end{array}$ & $\begin{array}{l}0.315 \\
0.24 \\
0.175 \\
0.15\end{array}$ & 50 \\
\hline & & 90 & $\begin{array}{l}0.7 \times 10^{12} \\
2.0 \times 10^{12} \\
3.7 \times 10^{12} \\
3.8 \times 10^{12}\end{array}$ & $\begin{array}{l}0.36 \\
0.204 \\
0.17 \\
0.132\end{array}$ & 30 \\
\hline
\end{tabular}
neutrons and the storage time $(t)$ of samples at the room temperature.

We also can suppose that the donor level $E_{v}+0.13 \mathrm{eV}$ that some authors attribute to a vacancy belongs to the Frenkel pair named $\mathrm{Si}_{\mathrm{FFCD}}$ (Fourfolded Coordinated Silicon Defect), with the lifetime of the order of hours at the room temperature [16]. The defect consists of a free vacancy and an interstitial silicon atom, which keeps bonds with the nearest neighbours, and at the same time raises the energy of vacancy donor level by $0.09 \mathrm{eV}$. The level $E_{v}+0.04 \mathrm{eV}$ can be observed by the EPR technique and cannot be detected by other methods. The level $E_{v}+0.13 \mathrm{eV}$ cannot be found by EPR and can be determined by the DLTS technique [24].

We can suppose that analogously to the divacancy $\left(\mathrm{V}_{2}\right)$ modified by carbon and oxygen [12] the presence of an interstitial silicon atom (I) results in increasing the donor level energy of free vacancy within a $\mathrm{Si}_{\mathrm{FFCD}}$ defect. In doing so, the acceptor level energy will be decreased by the same value $0.09 \mathrm{eV}$. Then, the acceptor level of $\mathrm{Si}_{\mathrm{FFCD}}^{(-/ 0)}$ defect will be located at $E_{c}-0.51 \mathrm{eV}$, and the acceptor level for $\mathrm{Si}_{\mathrm{FFCD}}^{(=-)}$defect will be $E_{c}-0.18 \mathrm{eV}$ in the forbidden gap of silicon.

\subsection{Isothermal annealing of $\mathrm{Si}\langle G e\rangle$}

It is known that the annealing leads to the recovery of solid characteristics. To study the kinetics of interaction of radiation defects, it is very important to determine their activation energy and frequency of jumps. To describe the annealing process, usually used are the equations of the chemical reaction kinetics [25]. It is supposed that the defect must obtain the energy $E_{a}$ to realize the reaction. At the temperature $T$, the fraction of such defects is defined by the exponential Boltzmann factor $\exp \left(-E_{a} / k T\right)$. If "fast" and "slow" components of annealing are present, the carrier concentration removed by defect clusters is described by the equation 


$$
\begin{aligned}
& n_{\mathrm{cl}}(t)=n_{01} \exp \left[-v_{1} t \exp \left(-\frac{E_{1}}{k T}\right)\right]+ \\
& +n_{02} \exp \left[-v_{2} t \exp \left(-\frac{E_{2}}{k T}\right)\right],
\end{aligned}
$$

where $\left(n_{01}+n_{02}\right)$ is the number of carriers removed by clusters from the conduction band after irradiation and that is returned into the conduction band under annihilation of trivacancies and divacancies with interstitial defects for the annealing time; $E_{1}$ and $E_{2}$ are the activation energies of the vacancy defect annealing by "fast" and "slow" components; $v_{1}$ and $v_{2}$ are their frequency factors (the jump number of defects per one second) of the annealing process; $t$ is the annealing duration; $k$ is the Boltzmann constant; $T$ is the annealing temperature.

The annealing process of defects can be investigated, determining $n_{\text {eff }}(T, \Phi)$ by the calculation of the carrier concentration removed both by point defects in the conducting matrix $n(T, \Phi)$ and by clusters $n_{\mathrm{cl}}(T, \Phi)$. The calculation of the $n_{\mathrm{cl}}$ dependence on the annealing time $(t)$ was carried out for the isothermal annealing of $\mathrm{Si}\langle\mathrm{Ge}\rangle$ samples irradiated by the fluence $\Phi$. The experimental data shown in Fig. 4 are well described by the equation of annealing kinetics (7). In the result of calculations of $n_{\mathrm{cl}}(t)$, according to Eq. (7), the following values were obtained: $E_{1}=0.74 \mathrm{eV}, v_{1}=$ $3.5 \times 10^{6} \mathrm{~s}^{-1}$ and $E_{2}=0.91 \mathrm{eV}, v_{2}=7 \times 10^{6} \mathrm{~s}^{-1}$. These parameters well describe the annealing at all three temperatures $(293,353$ and $379 \mathrm{~K})$. It should be noted that we used the term "annealing" for the description of changes of sample properties in the process of storage at the room temperature (see also [26]). This is correct in our case, because the storage time is ten times more than irradiation time and during the storage the carrier concentration in the conduction band is increased due to decreasing the defect cluster number. The recovery time of cluster charge is $\sim 0.1 \mathrm{~s}$ at the room temperature.

It is known $[26,27]$ that the concentration of Acentres increases under the annealing of silicon after hadrons irradiation. Since $V_{2}$ and $V_{3}$ are the main defects in clusters, therefore, the annealing of these defects leads to the vacancy generation $(\mathrm{V})$ and can be described by the following reactions:

$\mathrm{V}_{2}+\mathrm{I} \rightarrow \mathrm{V} ; \quad \mathrm{V}_{3}+\mathrm{I}_{2} \rightarrow \mathrm{V}$.

In this case, we supposed that vacancy type defects interact not only with interstitials (I), but also with diinterstitials $\left(\mathrm{I}_{2}\right)$.

Clusters of small sizes are annealed due to the mobility of vacancies at the room temperature. If the vacancy type defects are usually concentrated in clusters, then the interstitial type defects are concentrated in the conducting matrix. It is the high concentration of oxygen in studied $\mathrm{Cz}-\mathrm{Si}$ and $\mathrm{Si}\langle\mathrm{Ge}\rangle$ samples that leads to the increase of the interstitial defect concentration in the conducting volume. As shown in [12], oxygen prevents the exit of Si interstitial atoms onto the crystal surface and, probably, is the centre of di-interstitial defect formation. In [28], it was supposed that under a high density of interstitial atoms in the matrix they organize $\mathrm{Si}-\mathrm{Si}$ pairs. Such pairs are formed as a result of the stochastic location of two silicon atoms into the one interstitial space. These pairs are kept in one interstitial space only by the potential relief of crystal without formation of chemical covalent bonds between $\mathrm{Si}$ atoms.

We suppose that the annealing of clusters occurs at the direct annihilation of $\mathrm{I}$ and $\mathrm{I}_{2}$ with vacancy defects $\left(\mathrm{V}_{2}\right.$ and $\mathrm{V}_{3}$ ) according to the reactions (8). Then we attributed the migration energy $E_{1}=0.74 \mathrm{eV}$ and the frequency factor $v_{1}=3.5 \times 10^{6} \mathrm{~s}^{-1}$ to di-interstitials; $E_{2}=0.91 \mathrm{eV}$ and $v_{2}=7 \times 10^{6} \mathrm{~s}^{-1}-$ to silicon interstitial atoms. In the offered annealing model, we supposed that di-interstitials are more mobile (migration energy and frequency factor smaller) than interstitial atoms. The migration energy of oxygen dimers is $0.8 \mathrm{eV}$ less than that of single oxygen atom [29]. Moreover, according to [28], Si-Si diinterstitials are more mobile than the single Si interstitial atoms. In $[30,31]$, the values of migration energies of the interstitial atom and the vacancy are near $1 \mathrm{eV}$.

In [32] it was shown that in the annealing process the increase of cluster sizes occurs. It can be supposed that sizes of larger clusters grow when smaller clusters decay. This process can be described by the following equation

$$
\begin{aligned}
& n_{\mathrm{cl}}(t)=n_{01} \exp \left[-v t_{1} \exp \left(-\frac{E}{k T_{1}}\right)\right]+ \\
& +n_{02}\left[1-\exp \left(-v t_{1} \exp \left(-\frac{E_{\mathrm{V}}}{k T_{1}}\right)\right)\right]
\end{aligned}
$$

where $n_{01}$ is the number of carriers returned into the conduction band at the annealing of small clusters; $n_{02}$ is the number of carriers captured from the conduction band by large clusters.

The first term in (9) describes the annealing of small clusters due to the annihilation of interstitial atoms and di-interstitials with vacancy type defects according to the above reactions (8). As a result, the vacancies are released and other clusters can trap them. The number of removed carriers is increased and this process is described by the second term in (9). Using this model of the annealing, we obtain the value $E_{v}=0.8 \mathrm{eV}$ for the migration energy of vacancy and the frequency factor $v=1 \times 10^{7} \mathrm{~s}^{-1}$. The obtained migration energy of interstitial type defects $(E=0.795 \mathrm{eV})$ is equal to the average value of activation energies, namely 0.91 and $0.74 \mathrm{eV}$, used under the improved description of the annealing, according to the reactions (8). The frequency factor $(v)$ can be considered as a sum of frequency factors, namely $3.5 \times 10^{6}$ and $7 \times 10^{6} \mathrm{~s}^{-1}$, obtained in the same process (7). For such description, it is supposed that vacancies, released from the cluster, are captured by other clusters, rather than by impurities (for example, by oxygen), since the capture radius for impurities is near $5 \AA$ and several hundreds of angstrom for clusters. 


\section{Conclusions}

It was shown that doping with germanium $\left(N_{\mathrm{Ge}}=\right.$ $=2 \times 10^{20} \mathrm{~cm}^{-3}$ ) the Czochralski grown $n$-type silicon increases the radiation hardness of material. This is caused by both the decrease of the average radius of defect clusters (from $70 \AA$ in standard $\mathrm{Cz}-\mathrm{Si}$ to $40 \AA$ in $\mathrm{Si}\langle\mathrm{Ge}\rangle)$ and the reduction of introduction rate for divacancies $\left(E_{c}-0.42 \mathrm{eV}\right)$ and trivacancies $\left(E_{c}-0.49 \mathrm{eV}\right)$ into the conducting matrix of $\mathrm{Si}\langle\mathrm{Ge}\rangle$ (about 13 times). It is supposed that germanium in silicon is the recombination centre for the Frenkel pairs that suppresses the introduction of vacancy type defects. It was proposed that the decrease of defect cluster radius was caused by the germanium influence on the annealing of defects in clusters.

In the assumption that the acceptor level of $E_{c}-0.076 \pm 0.002 \mathrm{eV}$ belongs to $\mathrm{C}_{2 i} \mathrm{O}_{2 i}$ defect, it was shown that $\mathrm{C}_{i} \mathrm{C}_{s}$ defects are modified by oxygen dimers with the energy levels of $E_{c}-0.133 \mathrm{eV}$ and $E_{c}-0.093 \mathrm{eV}$ in oxygen-containing $n$-Si $\langle\mathrm{Ge}\rangle$.

It was proved that the $E_{c}-0.204 \mathrm{eV}$ level belongs to the A-centre modified by the interstitial silicon atom.

It was shown that the annealing of defect clusters after irradiation of $\mathrm{Si}\langle\mathrm{Ge}\rangle$ by fast neutrons is caused by annihilation of vacancy type defects with interstitial atoms and di-interstitials. The migration energy and frequency factor for di-interstitial $\left(E_{1}=0.74 \mathrm{eV}\right.$ and $v_{1}=$ $\left.3.5 \times 10^{6} \mathrm{~s}^{-1}\right)$, interstitial silicon atom $\left(E_{2}=0.91 \mathrm{eV}\right.$ and $\left.v_{2}=7 \times 10^{6} \mathrm{~s}^{-1}\right)$ and vacancy $\left(E_{v}=0.8 \mathrm{eV}, v=1 \times 10^{7} \mathrm{~s}^{-1}\right)$ were determined.

\section{References}

1. B.E. Kustov, M.G. Milvidskii, Yu.G. Semenov, B.M. Turovskii, V.I. Shakhovtsov, V.L. Shindich, Deformation charges of isovalent impurities in silicon // Fizika tekhnika poluprovodnikov 20 (2), p. 270-274 (1986) (in Russian).

2. L.I. Khirunenko, V.I. Shakhovtsov, V.V. Shumov, The radiation defect formation in Ge-doped silicon under low-temperature irradiation // Fizika tekhika poluprovodnikov 32 (2), p. 132-134 (1998) (in Russian).

3. G.D. Watkins // IEEE Trans. Nucl. Sci. NS-16 (6), p. 13-18 (1969).

4. C.V. Budtz-Jorgensen, P. Kringhoj, A. Nylandsted Larsen, N.V. Abrosimov, Deep-level transient spectroscopy of the Ge-vacancy pair in Ge-doped n-type silicon // Phys. Rev. B 58 (3), p. 1110-1113 (1998).

5. Yu.V. Pomozov, M.G. Sosnin, L.I. Khirunenko, V.I. Yashnik, N.V. Abrosimov, W. Shroder, M. Hohne, Oxygen-related radiation defects in $\mathrm{Si}_{1-\mathrm{x}} \mathrm{Ge}_{\mathrm{x}} / /$ Fizika tekhnika poluprovodnikov 34 (9), p. 1030-1034 (2000) (in Russian).

6. L.I. Khirunenko, V.I. Shakhovtsov, V.K. Shinkarenko, L.I. Shpinar, I.I. Yaskovets, Particularities of the processes of the radiation defect formation in $\mathrm{Si}\langle\mathrm{Ge}\rangle$ crystals // Fizika tekhnika poluprovodn. 21 (3), p. 562-565 (1987) (in Russian).

7. A.P. Dolgolenko, I.I. Fishchuk, Defect clusters and simple defect build-up kinetics in fast-neutron irradiated n-Si // Phys. status solidi (a) 50, p. 751755 (1978).

8. M.W. Thompson, Defects and radiation damage in metals. Mir, Moscow, 1971 (in Russian).

9. B.R. Gossick, Disordered regions in semiconductors bombarded by fast neutrons // J. Appl. Phys. 30 (8), p. 1214-1218 (1959).

10. A.P. Dolgolenko, I.I. Fishchuk, A-centres build-up kinetics in the conductive matrix of pulled n-type silicon with calculation of their recharges at defect clusters // Phys. status solidi (a) 67 (8), p. 407-411 (1981).

11. A.P. Dolgolenko, Variation of carrier removal rate with irradiation dose in fast-pile neutron irradiated n-Si // Phys. status solidi (a) 179, p. 179-188 (2000).

12. A.P. Dolgolenko, P.G. Litovchenko, M.D. Varentsov, G.P. Gaidar, A.P. Litovchenko, Particularities of the formation of radiation defects in silicon with low and high concentration of oxygen // Phys. status solidi (b), 243 (8), p. 1842-1852 (2006).

13. A.P. Dolgolenko, P.G. Litovchenko, A.P. Litovchenko, M.D. Varentsov, V.F. Lastovetsky, G.P. Gaidar, Influence of growing and doping methods on radiation hardness of n-Si irradiated by fast-pile neutrons // Semiconductor Physics, Quantum Electronics \& Optoelectronics 7 (1), p. 815 (2004).

14. M. Huhtinen, Simulation of non-ionising energy loss and defect formation in silicon // Nucl. Instrum. Methods A 491, p. 194-215 (2002).

15. P.G. Litovchenko, F. Lemeilleur, A.P. Dolgolenko, L.I. Barabash, N.N. Kolychev, A.P. Litovchenko, V.F. Lastovezky, T.I. Kibkalo, L.A. Polivtsev, Dose dependence of the concentration of carriers in high resistivity $\mathrm{Si}$ irradiated by $24 \mathrm{GeV}$ protons and properties of the detectors on its base // in: $3^{\text {rd }}$ ROSE Workshop on Radiation Hardening of Silicon Detectors, DESY, Hamburg, 12-14, February, 1998, p. 20-24.

16. S. Goedecker, Th. Deutsch, L. Billard, A fourfold coordinated point defect in silicon // Phys. Rev. Lett. 88 (23), p. $235501-235505$ (2002).

17. G.D. Watkins, J.W. Corbett, Defects in irradiated silicon. I. Electron spin resonance of the $\mathrm{Si} A$ center // Phys. Rev. 121, p. 1001-1014 (1961).

18. J.L. Lindstrom, T. Hallberg, J. Hermansson, L.I. Murin, B.A. Komarov, V.P. Markevich, M. Kleverman, B.G. Svensson, Interaction between self-interstitials and the oxygen dimer in silicon // Physica B 308-310, p. 284-289 (2001).

19. A. Dolgolenko, M. Varentsov, G. Gaidar, Energylevel position of bistable $\left(\mathrm{C}_{\mathrm{i}} \mathrm{C}_{\mathrm{s}}\right)^{0}$ defect in the $\mathrm{B}$ 
configuration in the forbidden band of $\mathrm{n}-\mathrm{Si} / /$ Phys. status solidi (b) 241, p. 2914-2922 (2004).

20. M. Asghar, M. Zafar Iqbai, N. Zafar, Characterization of deep levels introduced by alpha radiation in n-type silicon // J. Appl. Phys. 73 (8), p. 3698-3708 (1993).

21. G.E. Jellison, Jr, Transient capacitance studies of an electron trap at $E_{c}-E_{T}=0.105 \mathrm{eV}$ in phosphorusdoped silicon // J. Appl. Phys. 53 (8) p. 5715-5719 (1982).

22. P. Pellegrino, P. Leveque, J. Lavita, A. Hallen, C. Jagadish, B. G. Svensson, Annealing kinetics of vacancy-related defects in low-dose MeV self-ionimplanted n-type silicon // Phys. Rev. B 64, p. 195211-10 (2001).

23. J.L. Lindstrom, L.I. Murin, B.G. Svensson, V.P. Markevich, T. Hallberg, The $\mathrm{VO}_{2} *$ defect in silicon // Physica B 340-342, p. 509-513 (2003).

24. G. D. Watkins, J. R. Troxell, A. P. Chatterjee, Vacancies and interstitials in silicon // in: Intern. Conf. on Defects and Radiation Effects in Semiconductors, Nice, 1978. Inst. of Phys., Conf. Series, Number 46, 1979, p. 16-30.

25. R.F. Konopleva, V.N. Ostroumov, The interaction of high-energy charged particles with germanium and silicon. Atomizdat, Moscow, 1975 (in Russian).
26. M. Moll, E. Fretwurst, M. Kuhnke, G. Lindstrom, Relation between microscopic defects and macroscopic changes in silicon detector properties after hadrons irradiation // Nucl. Instrum. Methods $B$ 186, p. 100-110 (2002).

27. R.E. Whan, Oxygen-defect complexes in neutronirradiated silicon // J. Appl. Phys. 37 (9), p. 33783382 (1966).

28. A.R. Chelyadinskii, F.F. Komarov, The defectimpurity engineering in the implanted silicon // Uspekhi fizicheskikh nauk, 173 (8), p. 813-845 (2003) (in Russian).

29. S.J. Watts, C. Da Via, A. Karpenko, Macroscopic results for a novel oxygenated silicon material // Nucl. Instrum. and Meth. in Phys. Res. A, 485, p. 153-158 (2002).

30. S. Libertino, S. Coffa, Room temperature point defect migration in crystalline Si // Solid State Phenomena 82-84, p. 207-212 (2002).

31. C. Bonafos, D. Mathiot, A. Claverie, Ostwald ripening of end-of-range defects in silicon // J. Appl. Phys. 83 (6), p. 3008-3017 (1998).

32. J.M. Pankratz, J.A. Sprague, M.L. Rudee, Investigation of neutron-irradiation damage in silicon by transmission electron microscopy // $\mathrm{J}$. Appl. Phys. 39 (1), p. 101-106 (1968). 